\title{
Supporting Information \\ Predicting the Affinity of Peptides to Major Histocompatibility Complex Class II by Scoring Molecular Dynamics Simulations
}

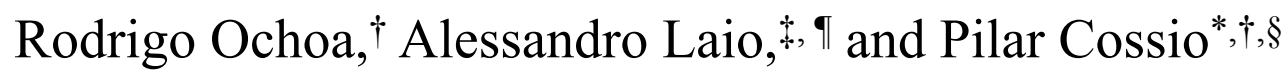

${ }^{\dagger}$ Biophysics of Tropical Diseases, Max Planck Tandem Group, University of Antioquia, 050010 Medellin, Colombia

International School for Advanced Studies (SISSA), Via Bonomea 265, 34136 Trieste, Italy

ฯ The Abdus Salam International Centre for Theoretical Physics (ICTP), Strada Costiera 11, 34151 Trieste, Italy

$\S$ Department of Theoretical Biophysics, Max Planck Institute of Biophysics, 60438

Frankfurt am Main, Germany

E-mail: *pilar.cossio@biophys.mpg.de,grupotandem.biotd@udea.edu.co 


\section{Supporting Figures}

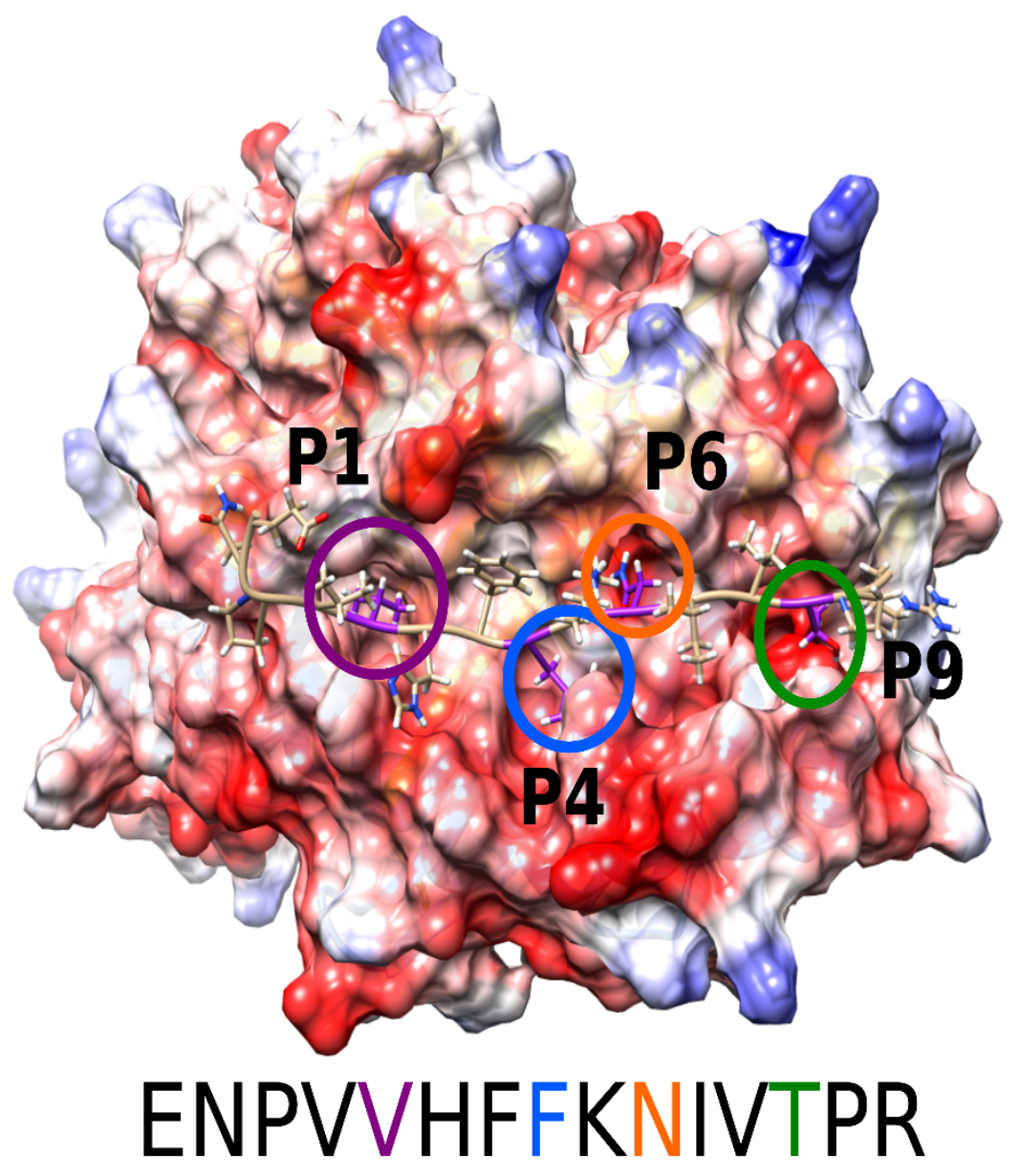

Figure S1. MHC class II bound to a peptide, which sequence is shown in bold. The PDB structure id is $1 \mathrm{BX} 2$. The interface is characterized by the key pockets $\mathrm{P} 1$ (purple circle), P4 (blue circle), P6 (orange circle) and P9 (green circle). The peptide amino acids interacting with the receptor pockets are colored in violet. 


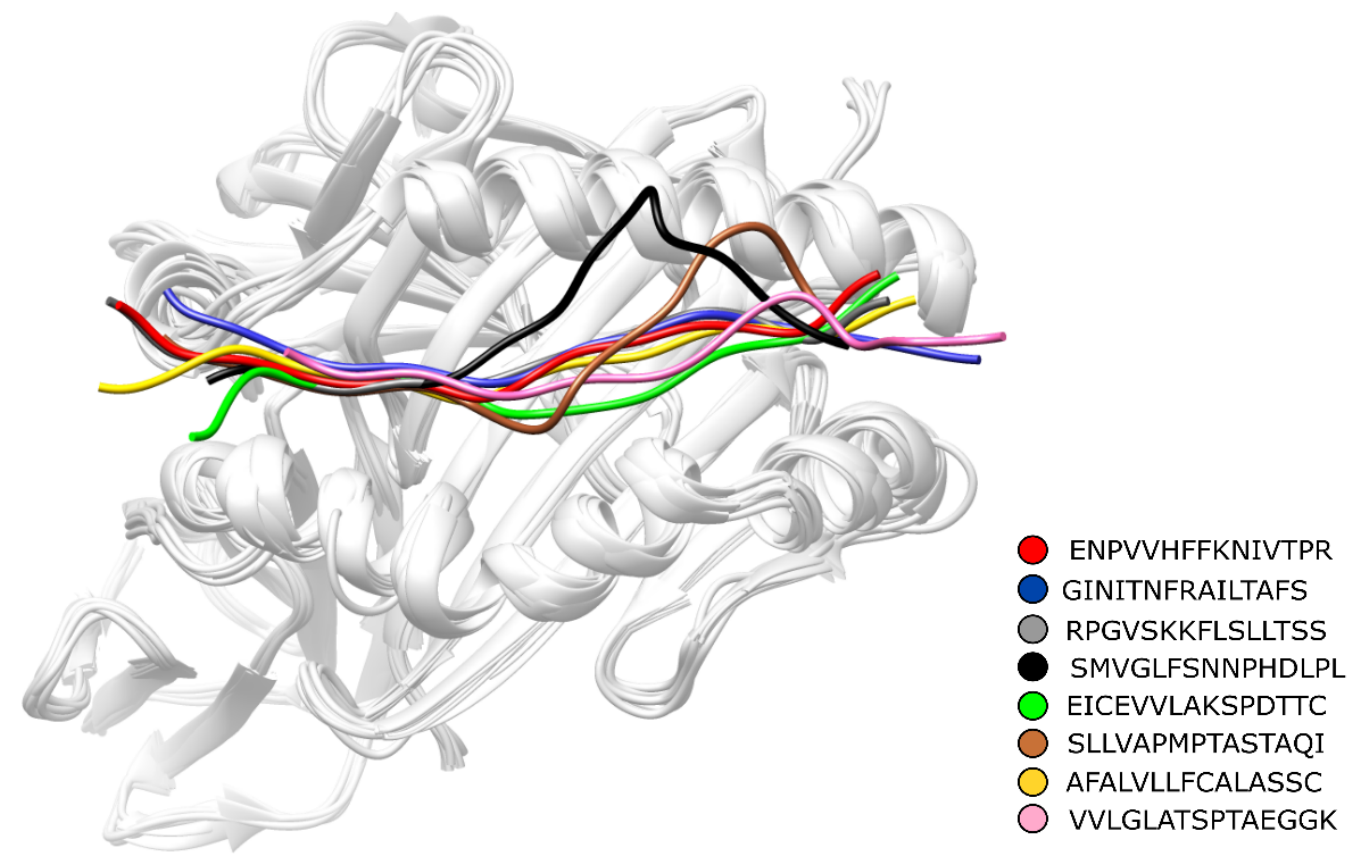

Figure S2. Conformations of the peptides bound to the MHC class II after $100 \mathrm{~ns}$ of MD simulations. The final conformation of some peptides (i.e. black and brown) shows a tendency of the peptide to partially detach from the binding groove. However, this region fluctuates during the simulated time according to the observables calculated over the trajectory (Figure S3). 
RMSD of peptide core region
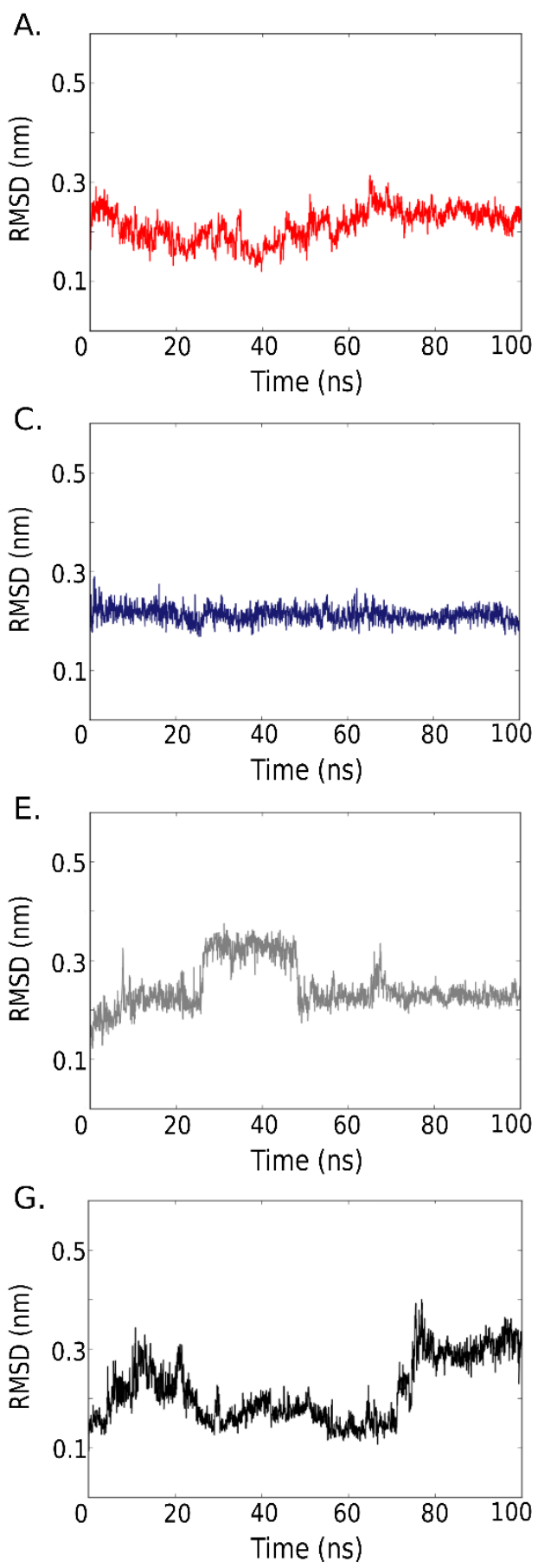

Hydrogen bonds
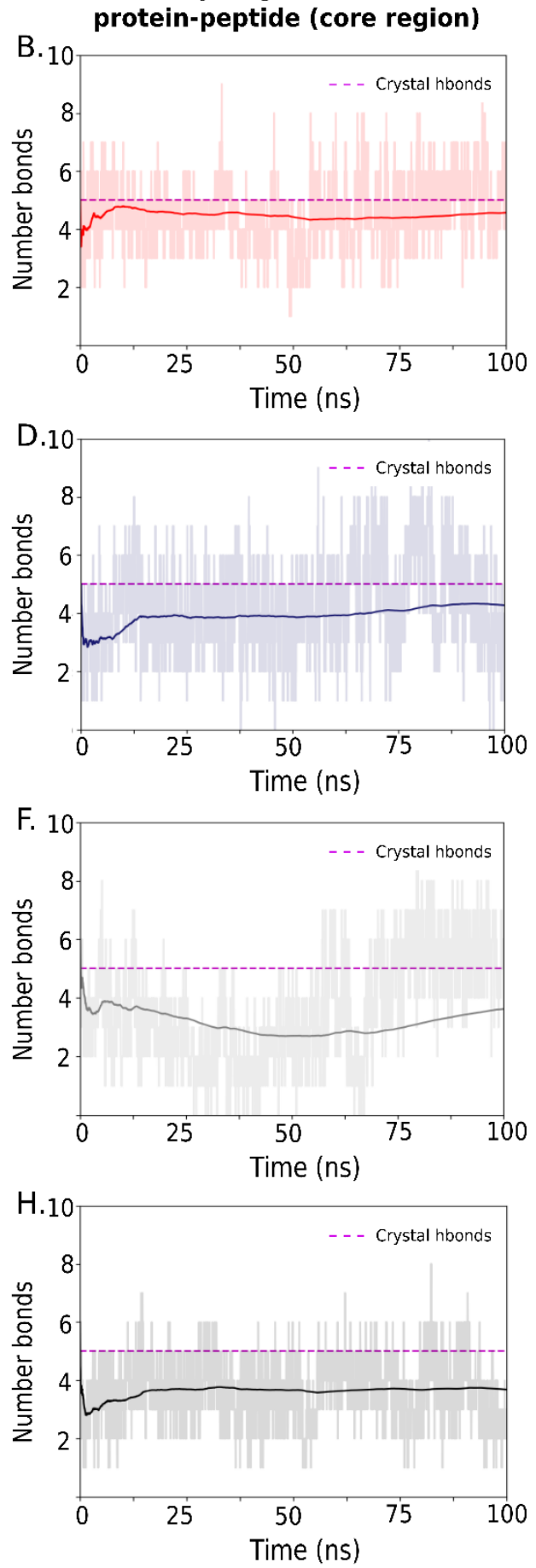

Figure S3. Observables that monitor the convergence of the peptide-MHC class II MD simulations. All-atom RMSD of the peptide during the $100 \mathrm{~ns}$ (left panels) and the number of hydrogen bonds (right panels) between the peptide and the protein during the trajectory. 
These observables are shown for peptides ENPVVHFFKNIVTPR (A and B), GINITNFRAILTAFS ( $\mathrm{C}$ and D), RPGVSKKFLSLLTSS (E and F) and SMVGLFSNNPHDLPL ( $G$ and $H$ ), respectively. The number of hydrogen bonds for the starting model is shown as a dashed magenta line in the right panels. 
RMSD of peptide core region

A.

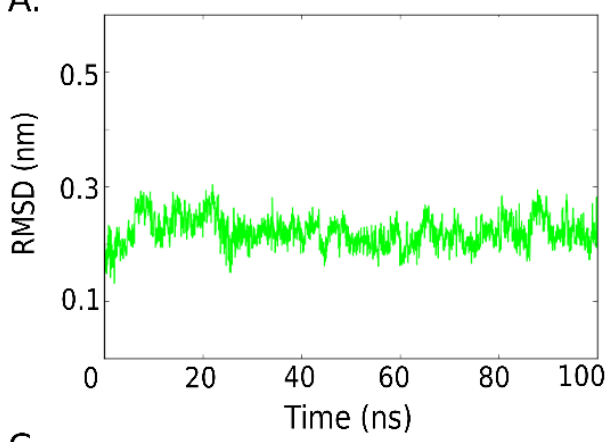

C.

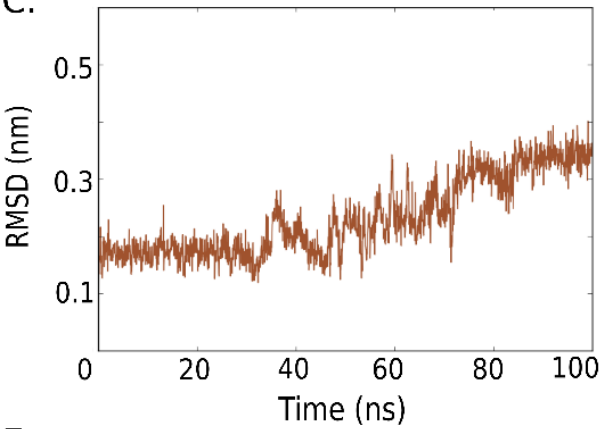

E.

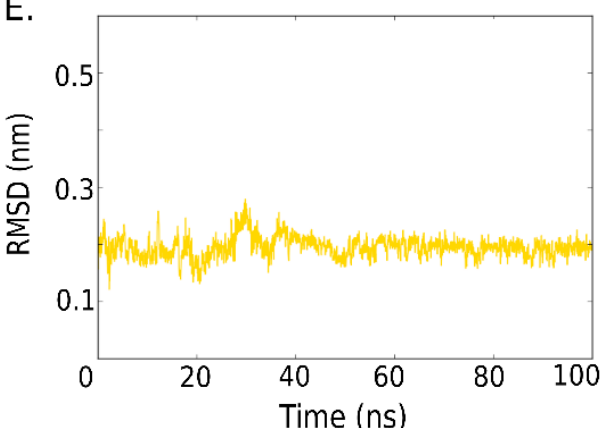

G.

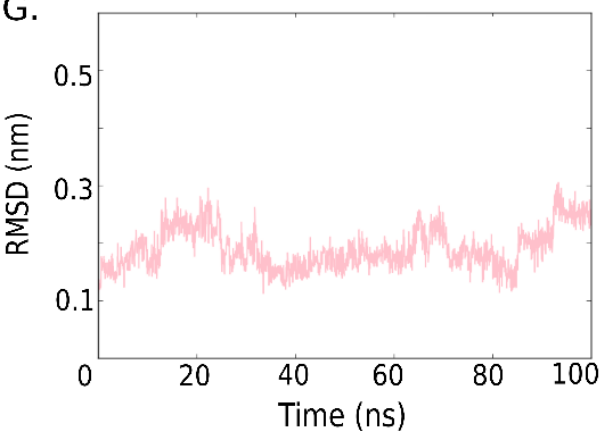

Hydrogen bonds
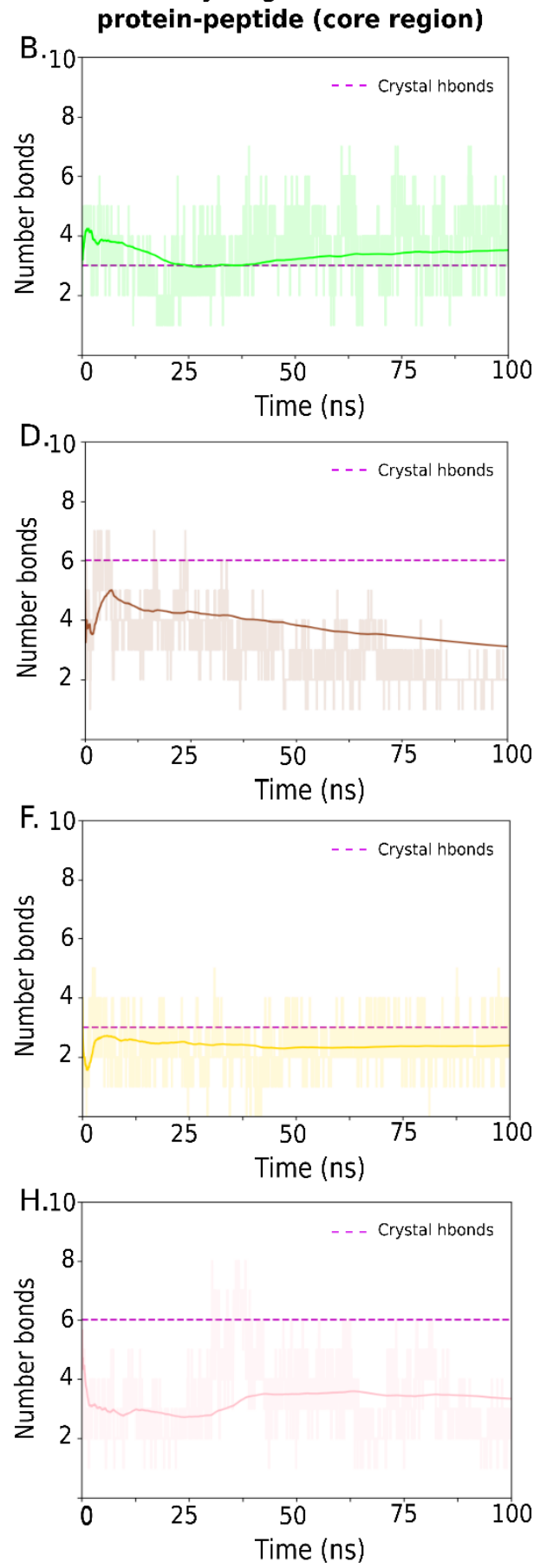

Figure S4. Observables that monitor the convergence of the peptide-MHC class II MD simulations. All-atom RMSD of the peptide during the $100 \mathrm{~ns}$ (left panels) and the number of hydrogen bonds (right panels) between the peptide and the protein during the trajectory. These observables are shown for peptides EICEVVLAKSPDTTC (A and B), 
SLLVAPMPTASTAQI ( $C$ and D), AFALVLLFCALASSC ( $E$ and $F$ ) and VVLGLATSPTAEGGK ( $\mathrm{G}$ and $\mathrm{H}$ ), respectively. The number of hydrogen bonds for the starting model is shown as a dashed magenta line in the right panel.
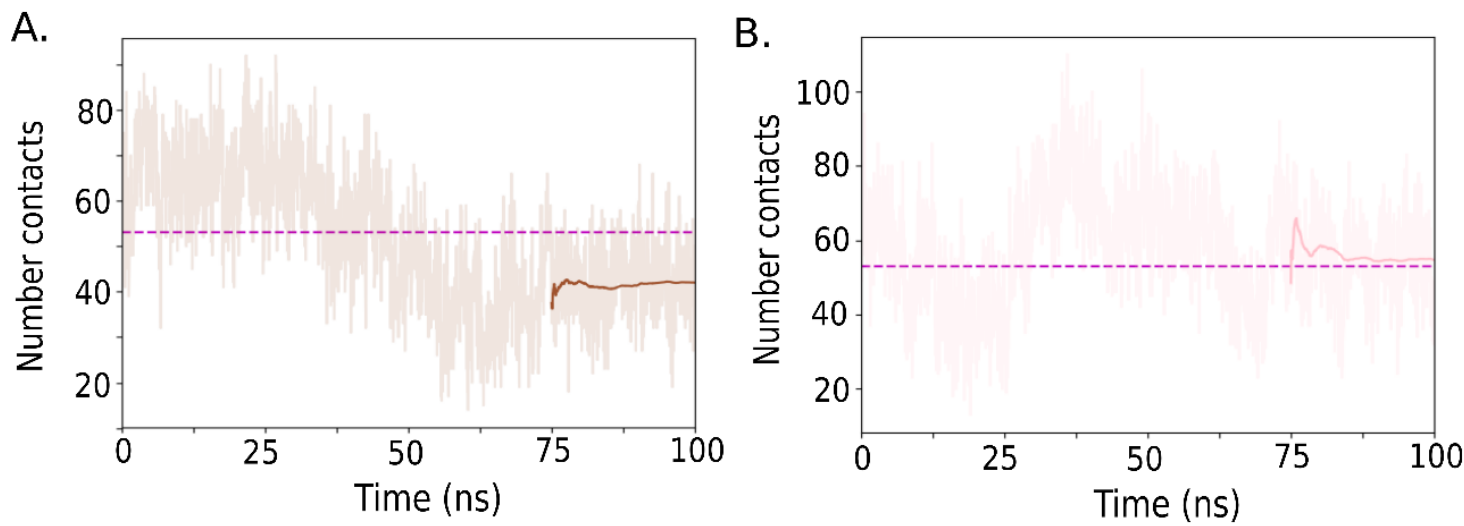

Figure S5. Number of contacts between the peptide and the protein during the trajectory. These observables are shown for peptides SLLVAPMPTASTAQI (A) and VVLGLATSPTAEGGK (B), respectively. A running average with time-window of $2.5 \mathrm{~ns}$ is shown for the last quarter of the trajectory ( $25 \mathrm{~ns})$ as a continuous line. The number of contacts for the starting model is shown as a dashed magenta line in the right panel. 
A.

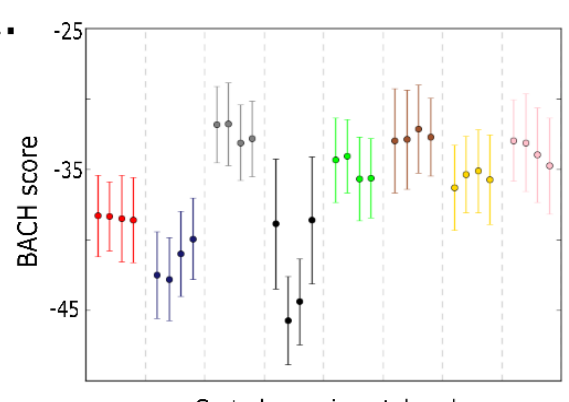

Sorted experimental rank

C.

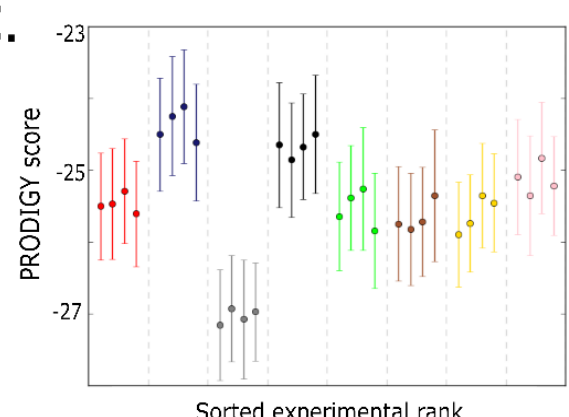

E.

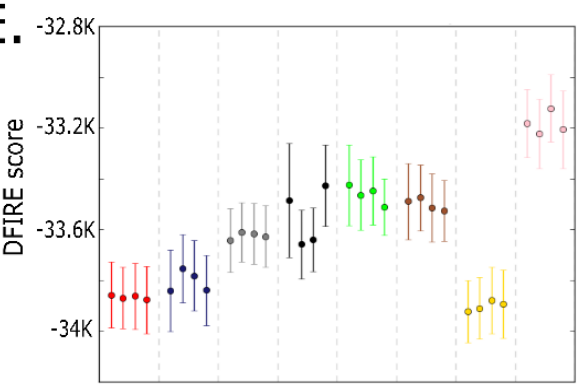

Sorted experimental rank

G.

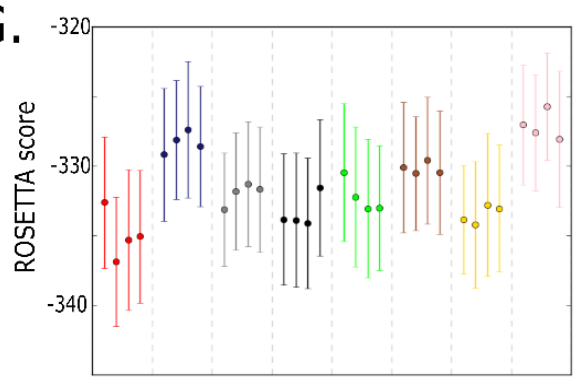

Sorted experimental rank
B.

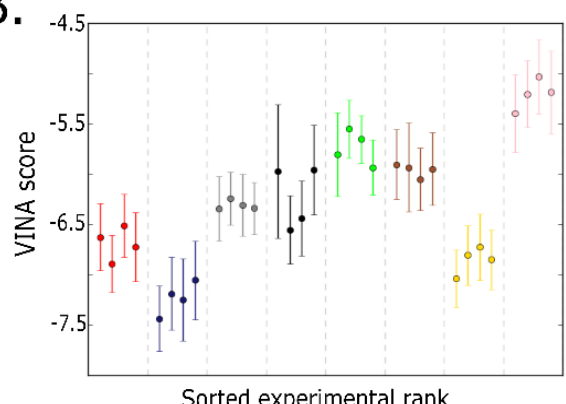

D.

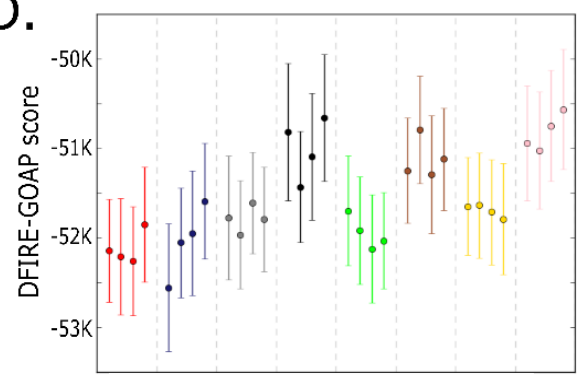

Sorted experimental rank

F.

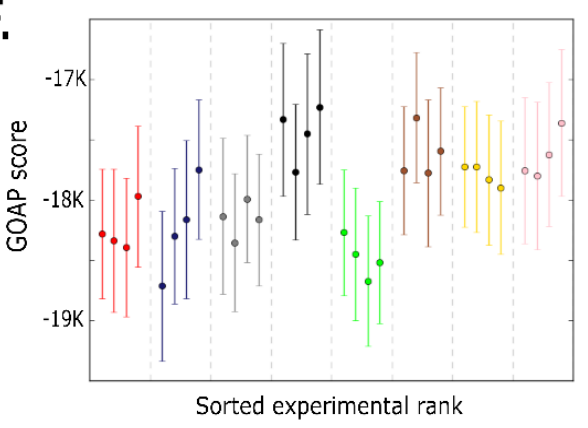

Sorted experimental rank
ENPVVHFFKNIVTPR GINITNFRAILTAFS RPGVSKKFLSLLTSS SMVGLFSNNPHDLPL EICEVVLAKSPDTTC SLLVAPMPTASTAQI AFALVLLFCALASSC

VVLGLATSPTAEGGK

Figure S6. Block analysis for the scoring functions BACH (A), VINA (B), PRODIGY (C), DFIRE-GOAP (D), DFIRE (E), GOAP (F) and ROSETTA (G) during the last quarter of the trajectory at $350 \mathrm{~K}$. The peptides are sorted from the highest activity (left) to the lowest (right). Each peptide is labeled by a color, and the blocks are represented by the average score and the standard deviation. 
A.

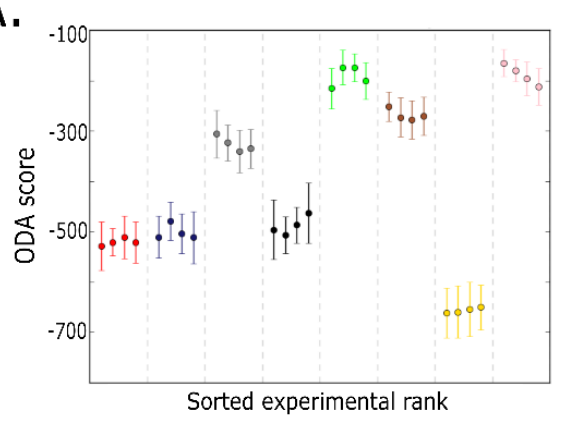

C.

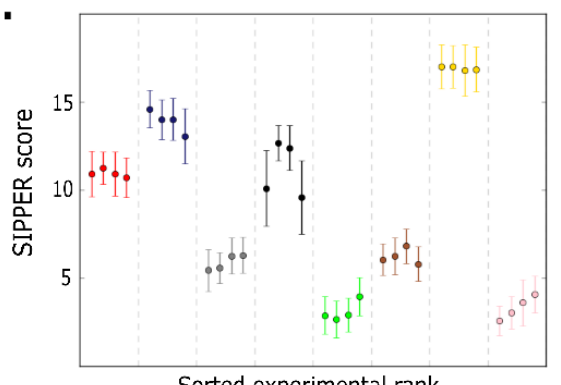

Sorted experimental rank

E.

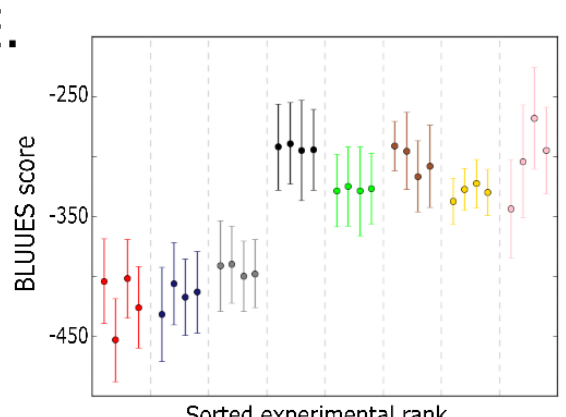

G.

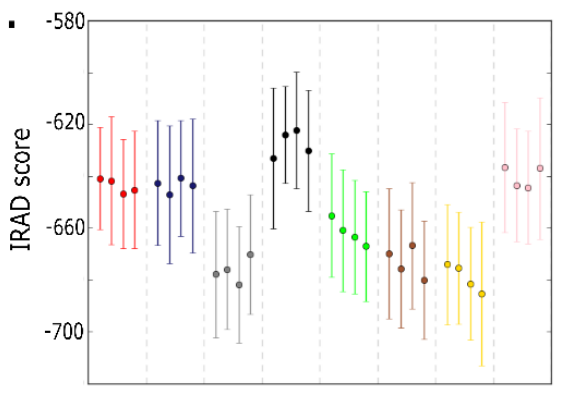

B.

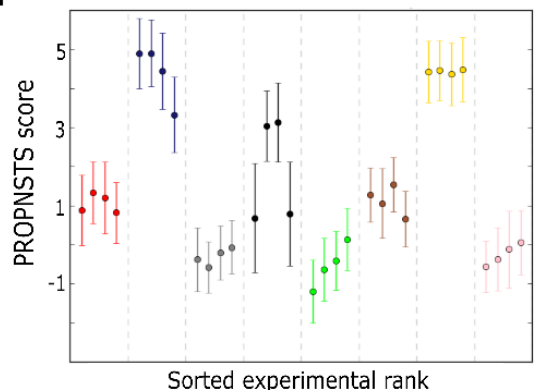

D.

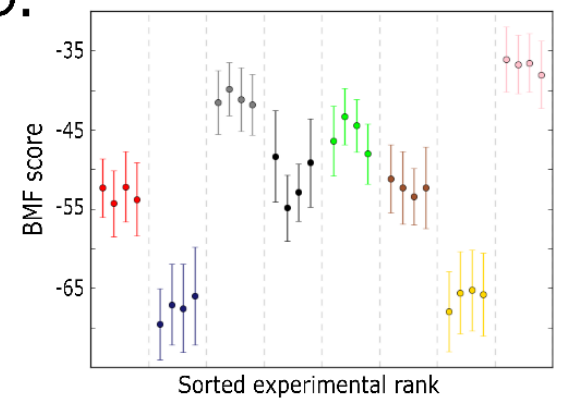

F.

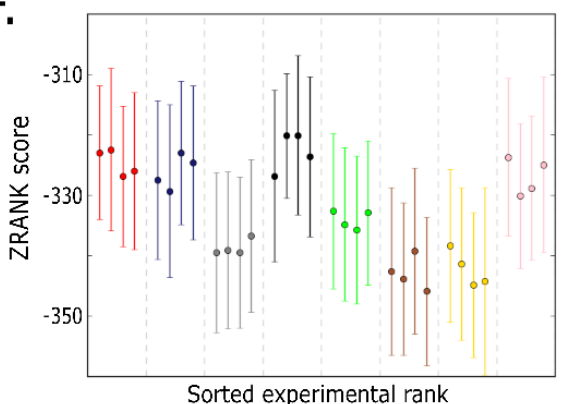

Sorted experimental rank

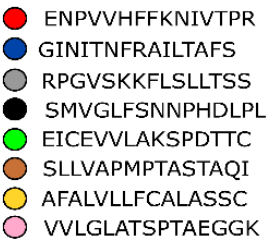

Sorted experimental rank

Figure S7. Block analysis for the scoring functions ODA (A), PROPNSTS (B), SIPPER (C), BMF (D), BLUUES (E), ZRANK (F) and IRAD (G) during the last quarter of the trajectory at $350 \mathrm{~K}$. The peptides are sorted from the highest activity (left) to the lowest 
(right). Each peptide is labeled by a color, and the blocks are represented by the average score and the standard deviation.

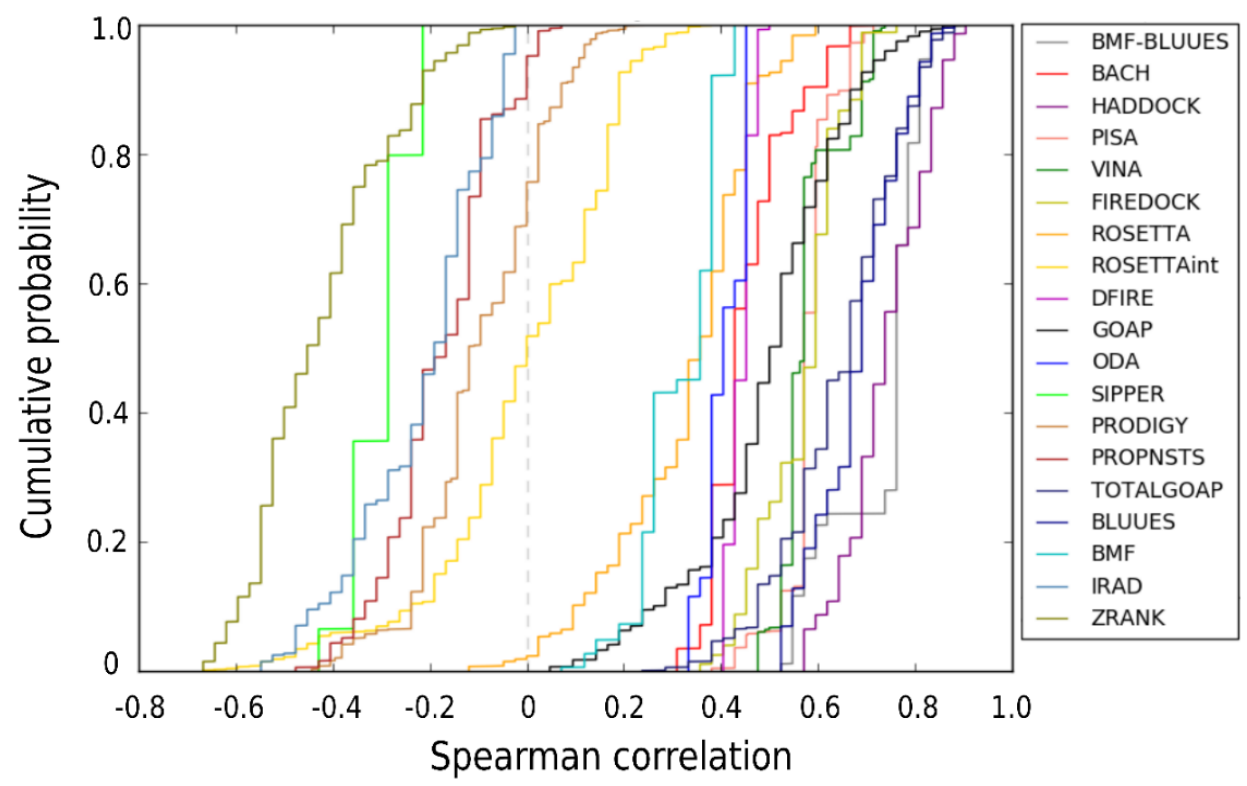

Figure S8. Cumulative probability distributions of Spearman correlations calculated after bootstrapping with 2000 replica for all the implemented scoring functions during the last quarter of the trajectory at $350 \mathrm{~K}$.

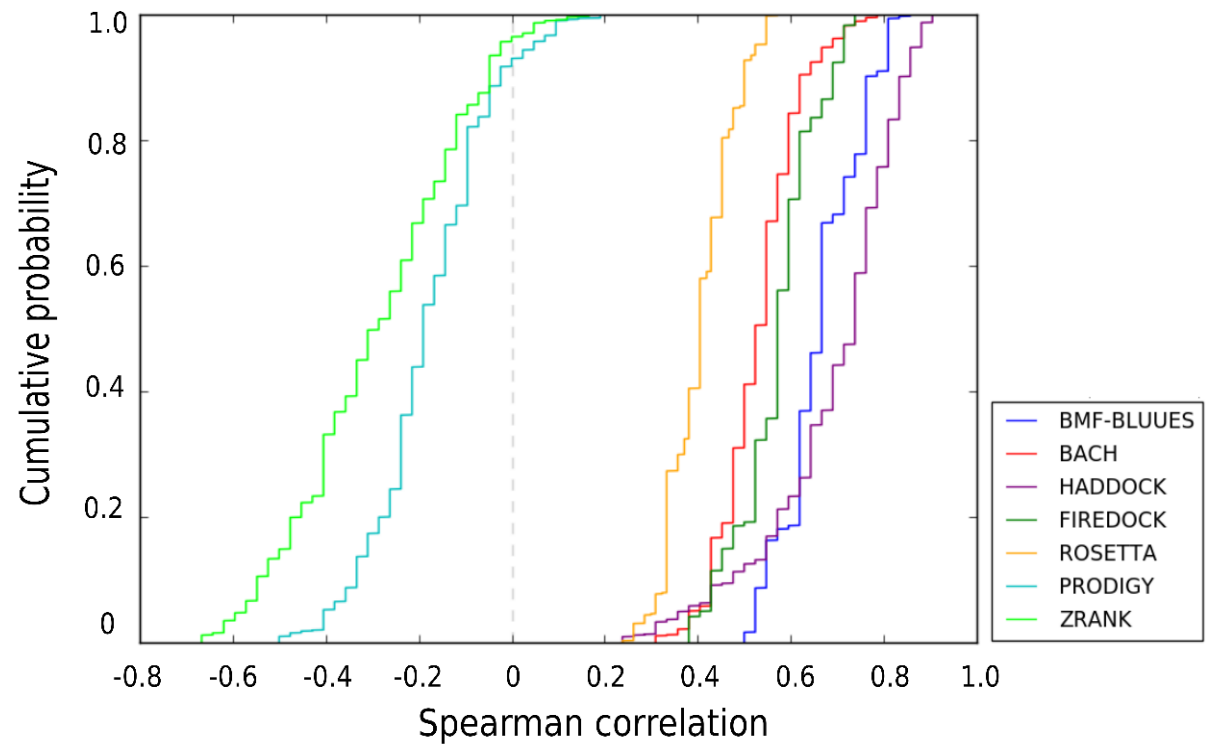


Figure S9. Cumulative probability distributions of Spearman correlations using half of the MD trajectory at $350 \mathrm{~K}$ and calculated after bootstrapping with 2000 replica for all the scoring functions BACH, HADDOCK, FIREDOCK, ROSETTA, PRODIGY, ZRANK and the combination BMF-BLUUES.

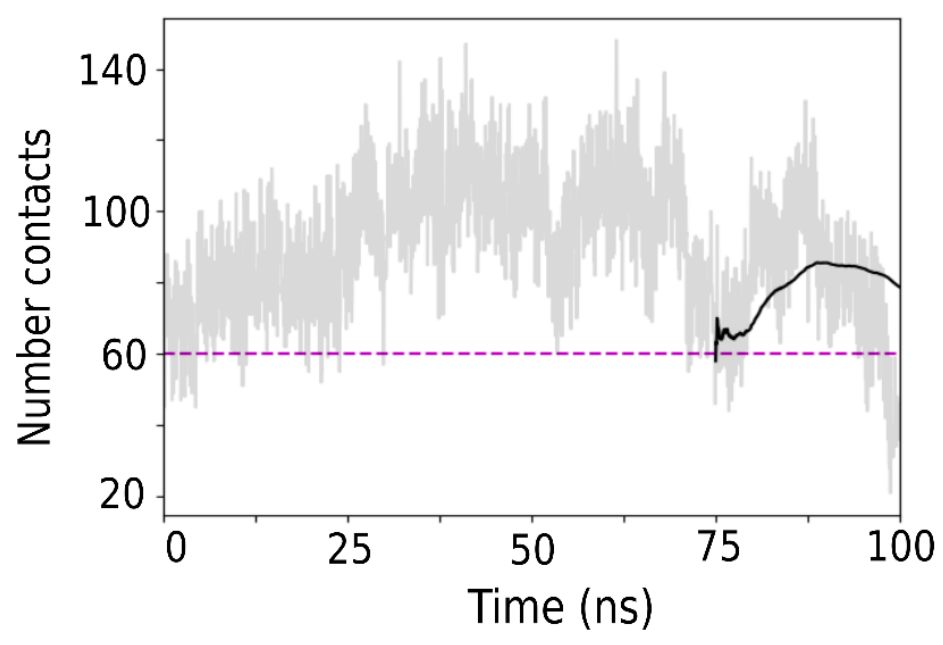

Figure S10. Number of contacts between the peptide SMVGLFSNNPHDLPL and the protein during the trajectory. We can note that the peptide has not completely converged. A running average with time-window of $2.5 \mathrm{~ns}$ is shown for the last quarter of the trajectory (25 ns) as a continuous line. The number of contacts for the starting model is shown as a dashed magenta line in the right panel. 


\section{Supporting Tables}

Table S1. Correlations between the results of the scoring functions: FIREDOCK, HADDOCK, PISA; BMF-BLUUES, DFIRE-GOAP and VINA for the pair-wise analysis. These scoring functions were implemented for the different consensus approaches. We note that HADDOCK is the least correlated to the rest.

\begin{tabular}{lllllll}
\hline $\begin{array}{l}\text { Scoring } \\
\text { functions }\end{array}$ & FIREDOCK & HADDOCK & PISA & $\begin{array}{l}\text { BMF- } \\
\text { BLUUES }\end{array}$ & $\begin{array}{l}\text { DFIRE- } \\
\text { GOAP }\end{array}$ & VINA \\
\hline FIREDOCK & 100.00 & & & & & \\
HADDOCK & 56.79 & 100.00 & & & & \\
PISA & 89.52 & 56.87 & 100.00 & & & \\
BMF-BLUUES & 76.33 & 72.10 & 74.57 & 100.00 & & \\
DFIRE-GOAP & 67.86 & 73.29 & 70.55 & 81.54 & 100.00 & \\
VINA & 88.43 & 60.56 & 86.42 & 78.68 & 69.64 & 100.00 \\
\hline
\end{tabular}

Table S2. Weights associated to the scoring functions selected for the pairwise analysis using the logistic regression model for each peptide-pair subset. Each row corresponds to the subset of pairs made by the peptide with all the others.

\begin{tabular}{llllllll}
\hline Peptide used to test & $\begin{array}{l}\text { BMF- } \\
\text { BLUUES }\end{array}$ & $\begin{array}{l}\text { FIRE } \\
\text { DOCK }\end{array}$ & HADDOCK & PISA & VINA & $\begin{array}{l}\text { GFIRE- } \\
\text { GOAP }\end{array}$ \\
\hline ENPVVHFFKNIVTPR & 0.395 & 0.263 & 0.807 & -0.286 & 0.994 & 0.789 \\
GINITNFRAILTAFS & 0.360 & 0.351 & 1.289 & -0.166 & 0.608 & 0.651 \\
RPGVSKKFLSLLTSS & -0.315 & 0.575 & 0.961 & 0.575 & 0.172 & 1.151 \\
SMVGLFSNNPHDLPL & 1.132 & 0.275 & 0.562 & -0.508 & 0.170 & 1.752 \\
EICEVVLAKSPDTTC & 0.446 & 0.117 & 1.164 & 0.117 & 0.430 & 1.156
\end{tabular}




\begin{tabular}{lllllll} 
SLLVAPMPTASTAQI & 0.298 & 0.612 & 1.230 & -0.022 & 0.607 & 1.207 \\
AFALVLLFCALASSC & 0.454 & 0.599 & 0.922 & 0.083 & 1.327 & 1.056 \\
VVLGLATSPTAEGGK & 0.130 & 0.319 & 1.351 & -0.268 & 0.539 & 0.842 \\
& & & & & & \\
External test set & $\mathbf{0 . 2 5 5}$ & $\mathbf{0 . 4 4 5}$ & $\mathbf{1 . 2 4 1}$ & $\mathbf{- 0 . 1 7 9}$ & $\mathbf{0 . 7 4 3}$ & $\mathbf{1 . 1 4 5}$ \\
\hline
\end{tabular}

Table S3. Spearman correlation of $\Delta \Delta \mathrm{G}$ using the linear regression model. Because the model was trained to predict the sign (and not the exact score), for some peptides its performance is not ideal.

\begin{tabular}{ll}
\hline Peptide used to test & Spearman correlation $\mathbf{\Delta} \mathbf{\Delta G}$ - Linear model \\
\hline ENPVVHFFKNIVTPR & 0.697 \\
GINITNFRAILTAFS & 0.908 \\
RPGVSKKFLSLLTSS & 0.160 \\
SMVGLFSNNPHDLPL & 0.723 \\
EICEVVLAKSPDTTC & 0.429 \\
SLLVAPMPTASTAQI & 0.503 \\
AFALVLLFCALASSC & 0.200 \\
VVLGLATSPTAEGGK & 0.363 \\
\hline
\end{tabular}

\section{Supporting Text}

\section{Details of the scoring functions}

The scoring functions used in this study are mostly statistical and knowledge-based potentials used for protein-protein and protein-ligand docking. We also include some semiempirical approaches. All the scoring functions used in this work are open source. In the following, we describe the general details about their foundations. 
- ROSETTA (version 2016.32): Statistical method composed of multiple energy terms optimized for different kind of interactions, including protein-protein interactions. The all-atom terms describe the individual residue environments and frequent residue-pair interactions obtained from the Protein Data Bank (PDB). Additional terms account for packing of van der Waals spheres, hydrogen bonding and secondary structure elements. The score used was also optimized for docking calculations. ${ }^{1}$

- PISA (version 2011): Scoring function with an all-atom potential description. It is dependent on the interface area, the residue/atom composition and contacts, hydropathy index, charge distribution, topological complementarity, and other parameters. One of its main applications is to score crystal packing of biologically relevant structures. This scoring function is commonly used with PIE to rescore conformations, but in this work was used alone with the default parameters. ${ }^{2}$

- BMF (version 3): Set of empirical energies calculated using statistical information from ensembles of protein structures, reflecting the propensity of each amino acid to interact with others. The methodology has been applied for protein-fold recognition problems, and combined with other functions such as BLUUES (as applied in this work). ${ }^{3}$

- BLUUES (version 2): The method was developed to run analysis of electrostatic properties of proteins. It calculates the generalized Born radii, the electrostatic solvation free energy, the electrostatic forces on each atom, $\mathrm{pH}$-dependent properties, pKa of all ionizable groups, and the electrostatic potentials at the surface of the molecules and in a volume surrounding it. The methodology has been combined with other functions such as BMF (as applied in this work). ${ }^{4}$

- BMF-BLUUES: The arithmetic combination of the BMF and BLUUES outputs. 
- HADDOCK (version 2.1): Docking program with different options for the scoring function. The one implemented here is an energy estimation, computed as a weighted sum of different physical terms. These terms describe the Van der Waals interactions, electrostatic contributions, a set of interactions which use structural data to guide the potential, and a term that accounts for the desolvation energy relative to the interface area. For this project, a protocol provided by the developers to rescore protein-protein interactions was implemented. ${ }^{5}$

- VINA (version 1.1.2): Program derived from AutoDock, used mainly for docking of small molecules and small peptides against protein structures. The scoring function uses a semi-empirical approach, with a conformation-dependent part that includes the terms about repulsion, hydrophobic interactions, hydrogen bonding, and number of rotations. Despite that it is used with smaller ligands, its generalization lets us apply it for larger oligopeptides in bound conformations. ${ }^{6}$

- DFIRE: Energy function based on the orientation angles involved in dipole-dipole interactions. To achieve this, each polar atom is treated as a dipole, and its placement depends on the connection with other heavy atoms. The function is derived from protein structures, which also considers hydrogen-bonding interactions. The method has been mainly applied for protein folding, but can be combined with other methods such as GOAP (as applied in this work). ${ }^{7}$

- GOAP: All-atom statistical potential for the prediction of protein structures. The method relies on the planes associated to the interacting pairs created by each heavy atom. It is decomposed in distance- and angle-dependent contributions, integrating orientation-dependent polar atom interactions, hydrogen bonding and side chain interactions. The method can be combined with other methods such as DFIRE (as applied in this work). ${ }^{8}$ 
- DFIRE-GOAP: Arithmetic combination of the DFIRE and GOAP outputs, where DFIRE contributes with the distance-dependent terms, and GOAP with the angledependent. ${ }^{8}$

- ODA (version 3.2.3 of PyDock): Method for predicting protein-protein interaction sites. The algorithm generates protein surface patches of different sizes and obtains the optimal docking area based on atomic solvation parameters derived from octanol/water transfer experiments adapted for protein-protein binding. The method is part of a set of functions available in the PyDock docking program. ${ }^{9}$

- PROPNSTS (version 3.2.3 of PyDock): Part of the SIPPER scoring function that accounts for residue-residue interface propensities for the scoring of docking poses. The method was derived from a large set of curated heterodimeric complexes. ${ }^{10}$

- SIPPER (version 3.2.3 of PyDock): Full scoring function containing the outputs of ODA and PROPNSTS. The method integrates knowledge-based potentials with energy-based scoring protocols adapted to work at the residue level. ${ }^{10}$

- FIREDOCK: Docking program with a physical scoring function based on the estimation of binding free energies, solvation, electrostatics, van der Waals, hydrogen bonds, pi-stackings, rotamer torsion energies, aliphatic interactions, and the degree of exposure of the residues. The weights associated to each term are optimized using a machine learning approach. ${ }^{11}$

- ZRANK: Scoring function designed to rank structures predicted by the docking program ZDOCK. This considers a linear combination of atom energy terms weighted by optimized parameters. The terms include van der Waals attractive and 
repulsive terms, electrostatic attractive and repulsive, long- and short-range terms and an additional potential derived from monomeric protein structures. ${ }^{12}$

- IRAD: Update of the original ZRANK scoring function. The new version includes an additional four residue-based potentials, where three of them account for proteinprotein interactions, and one for protein folding. The implementation was applied with the complete peptide-protein complex. ${ }^{13}$

- PRODIGY (version 2015): Descriptor of protein-protein binding affinities based on the number of interfacial contacts at the interface. The terms have been combined with properties of the non-interacting surface. ${ }^{14}$

- BACH (version 6): Residue-wise knowledge-based potential that implements a Bayesian formalism, where the contributions account for interactions between amino acids and protein-solvent interactions. The contacts between amino acids include secondary structure specific contacts, Van der Waals contacts, among others. ${ }^{15,16}$ The program was run considering only the residues located at the interface between the peptide and receptor. ${ }^{17}$

\section{References}

(1) Alford, R. F.; Leaver-Fay, A.; Jeliazkov, J. R.; O’Meara, M. J.; DiMaio, F. P.; Park, H.; Pacella, M. S.; Bonneau, R.; Bradley, P.; Dunbrack, R. L.; Das, R.; Baker, D.; Kuhlman, B.; Kortemme, T.; Gray, J. J. The Rosetta All-Atom Energy Function for Macromolecular Modeling and Design. J. Chem. Theory Comput. 2017, 13, 3031-3048.

(2) Krissinel, E.; Henrick, K. Inference of Macromolecular Assemblies from Crystalline State. J. Mol. Biol. 2007, 372, 774-797.

(3) Berrera, M.; Molinari, H.; Fogolari, F. Amino Acid Empirical Contact Energy Definitions for Fold Recognition in the Space of Contact Maps. BMC Bioinformatics

2003, 4,8 . 
(4) Fogolari, F.; Corazza, A.; Yarra, V.; Jalaru, A.; Viglino, P.; Esposito, G. Bluues: a Program for the Analysis of the Electrostatic Properties of Proteins Based on Generalized Born Radii. BMC Bioinformatics 2012, 13, S18.

(5) Dominguez, C.; Boelens, R.; Bonvin, A. M. J. J. HADDOCK: A Protein-Protein Docking Approach Based on Biochemical or Biophysical Information. J. Am. Chem. Soc. 2003, 125, 1731-1737.

(6) Trott, O.; Olson, A. J. AutoDock Vina: Improving the Speed and Accuracy of Docking with a New Scoring Function, Efficient Optimization, and Multithreading. J. Comput. Chem. 2009, 31, 455-461.

(7) Yang, Y.; Zhou, Y. Specific Interactions for Ab Initio Folding of Protein Terminal Regions with Secondary Structures. Proteins: Struct. Funct. Genet. 2008, 72, 793-803.

(8) Zhou, H.; Skolnick, J. GOAP: A Generalized Orientation-dependent, All-atom Statistical Potential for Protein Structure Prediction. Biophys. J. 2011, 101, 2043-2052.

(9) Fernandez-Recio, J.; Totrov, M.; Skorodumov, C.; Abagyan, R. Optimal Docking Area: A New Method for Predicting Protein-Protein Interaction Sites. Proteins: Struct. Funct. Bioinf. 2004, 58, 134-143.

(10) Pons, C.; Talavera, D.; De La Cruz, X.; Orozco, M.; Fernandez-Recio, J. Scoring by Intermolecular Pairwise Propensities of Exposed Residues (SIPPER): A New Efficient Potential for Protein-Protein Docking. J. Chem. Inf. Model. 2011, 51, 370-377

(11) Andrusier, N.; Nussinov, R.; Wolfson, H. J. FireDock: Fast Interaction Refinement in Molecular Docking. Proteins: Struct. Funct. Bioinf. 2007, 69, 139-159.

(12) Pierce, B.; Weng, Z. ZRANK: Reranking Protein Docking Predictions with an Optimized Energy Function. Proteins: Struct. Funct. Bioinf. 2007, 67, 1078-1086.

(13) Vreven, T.; Hwang, H.; Weng, Z. Integrating Atom-based and Residue-based Scoring Functions for Protein-Protein Docking. Protein Sci. 2011, 20, 1576-1586.

(14) Xue, L. C.; Rodrigues, J. P.; Kastritis, P. L.; Bonvin, A. M.; Vangone, A. PRODIGY: A Web Server for Predicting the Binding Affinity of Protein-Protein Complexes. Bioinformatics 2016, 32, 3676-3678. 
(15) Cossio, P.; Granata, D.; Laio, A.; Seno, F.; Trovato, A. A Simple and Efficient Statistical Potential for Scoring Ensembles of Protein Structures. Sci. Rep. 2012, 2, 1-8.

(16) Sarti, E.; Zamuner, S.; Cossio, P.; Laio, A.; Seno, F.; Trovato, A. BACHSCORE. A Tool for Evaluating Efficiently and Reliably the Quality of Large Sets of Protein Structures. Computer Physics Communications 2013, 184, 2860-2865.

(17) Sarti, E.; Granata, D.; Seno, F.; Trovato, A.; Laio, A. Native Fold and Docking Pose Discrimination by the Same Residue-based Scoring Function. Proteins: Struct. Funct. Bioinf. 2015, 83, 621-630. 